\title{
Optopneumatic Detector with Platinum Flow Sensor
}

\author{
K. JASEK ${ }^{a, *}$, T. BUDZYŃSKI ${ }^{b}$ AND E. STRYSZAK ${ }^{a}$ \\ ${ }^{a}$ Military University of Technology, S. Kaliskiego 2, 00-908 Warsaw, Poland \\ ${ }^{b}$ Institute of Electron Technology, al. Lotników 32/46, 02-668 Warsaw, Poland
}

\begin{abstract}
In optopneumatic detectors, operating on principle of the gaseous infrared detectors, the radiation absorbed in the gas is changed into pressure fluctuation, followed by periodic flow of the gas between detector chambers. This flow is usually measured by means of hot-wire or hot-film anemometers. Their parameters in a great measure determine the quality of the whole detector. The flow sensor used in optopneumatic detectors should have short time constant, high sensitivity, possibility to distinguish the flow direction, and high chemical resistance to the gas which the detector is filled with. To meet these requirements, micro-electro-mechanical system flowmeter consisting of two platinum hot films placed in series in the flow direction was made. Platinum film of $0.3 \mu \mathrm{m}$ thickness was deposited by magnetron spattering on thin membrane, which was composed of polysilicon substrate $(0.5 \mu \mathrm{m})$ and two $\mathrm{Si}_{3} \mathrm{~N}_{4}$ layers with thickness of $0.1 \mu \mathrm{m}$. The obtained platinum elements have resistance of $32 \Omega$ and temperature coefficient of resistance of $2.31 \times 10^{-3} /{ }^{\circ} \mathrm{C}$. Hot film does not change its parameters up to $350^{\circ} \mathrm{C}$, whereas typical working temperature of such films ranged from 150 to $200^{\circ} \mathrm{C}$. Anemometer characteristics and voltage-current dependences were determined in this work. The linear range of operation from 0 to $10 \mathrm{ml} / \mathrm{min}$ and sensitivity suitable for optopneumatic detectors were found. The time constant of the flowmeter is equal to $11 \mathrm{~ms}$ (95\% of final signal), that enables the detector to operate with frequency up to $100 \mathrm{~Hz}$. The flowmeters were applied in detectors filled with sulphur dioxide and were tested for six months at temperature of hot-film about $150^{\circ} \mathrm{C}$. During that time no changes of their parameters were observed.
\end{abstract}

PACS numbers: 68.35.Iv, 07.07.Df

\section{Introduction}

Optoacoustic detectors, utilizing the phenomenon of the excitation of sound waves in gases by a modulated light beam, are commonly used as the detectors of infrared radiation. The amount of the radiation absorbed by a gas is usually measured by means of a microphone. These detectors are known as Golay chambers and used for the investigations of light absorption mechanism as well as for the trace analysis of gases and pollution monitoring [1-4]. Other varieties of gaseous detectors of infrared radiation are optopneumatic detectors in which the flow of gas between two chambers of the detector, instead of a periodic pressure change, is measured $[5,6]$. Their construction is schematically shown in Fig. 1.

A detector is composed of a metal housing 1 , divided into two chambers 5 and 7 filled with a gas absorbing infrared radiation. A front chamber closure 4 and a partition 6 separating two chambers are made of a material transparent to infrared radiation. The chambers are joined with channel 2 , in which a flow sensor 3 is placed.

After going through window 4 modulated infrared radiation first enters the front 5 and then the rear chamber 7 of an optopneumatic detector. Periodic absorption of infrared radiation by a gas filling in the chambers causes

\footnotetext{
* corresponding author; e-mail: kjasek@wat.edu.pl
}

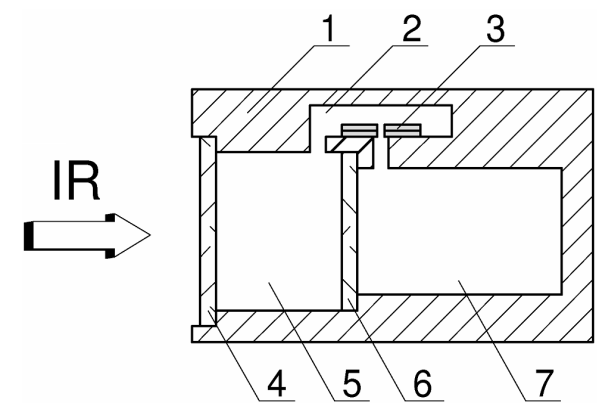

Fig. 1. The assembly of optopneumatic detector.

periodical heating of the gas accompanied by respective changes in pressure. The infrared radiation is being absorbed mainly in the front chamber and as a result of a pressure difference between the chambers a periodical flow of the gas appears, which is converted into an electrical signal by a flowmeter. The amplitude of this flow rate is a measure of the amount of radiation arriving at the detector within the absorption band of the gas.

The flowmeter utilized for the optopneumatic detectors should fulfil some conditions. In particular, it should be very small, show a small time constant and high sensitivity. It should also have an ability to distinguish flow direction and be resistant to chemical action of a gas filling the detector. Moreover, the flowmeter can disturb the 
flow and constitute a considerable resistance to the flow, which is unacceptable in other applications. Because of an oscillating character of the flow only the variable component of a signal from the flowmeter is used. Thus the change of the constant component of a signal (for example, being the result of the changes in the ambient temperature) is of low importance unlike the stability of the flowmeter sensitivity.

Thermal anemometers are used to measure flow in optopneumatic detectors, but there is no description of their design and parameters. In order to fulfil these specific requirements, thermal anemometer with platinum hot-film was designed, constructed and tested.

\section{The structure and operation of the flowmeter system}

The flowmeter contains two platinum elements in the form of conducting paths with rectangular intersection $65 \times 0.3 \mu \mathrm{m}^{2}$. These structures are placed on thin membrane, which is composed of polysilicon substrate $(0.5 \mu \mathrm{m})$ and two $\mathrm{Si}_{3} \mathrm{~N}_{4}$ layers with thickness of $0.1 \mu \mathrm{m}$ (Fig. 2). The membrane was prepared as follows: silicon nitride and polysilicon layers were deposited on a silicon wafer using low pressure chemical vapour deposition (LPCVD) method. The shape of the membrane was thus defined by means of photolithography. Then, the second layer of silicon nitride was deposited. In the next step thin titanium film $(0.02 \mu \mathrm{m})$, used as an adhesive layer, and platinum film were deposited by magnetron spattering and pattered. Finally, the membrane was released from its underlying silicon substrate by $\mathrm{KOH}$ etching. The membrane is $600 \times 600 \times 0.7 \mu \mathrm{m}^{3}$ in size.

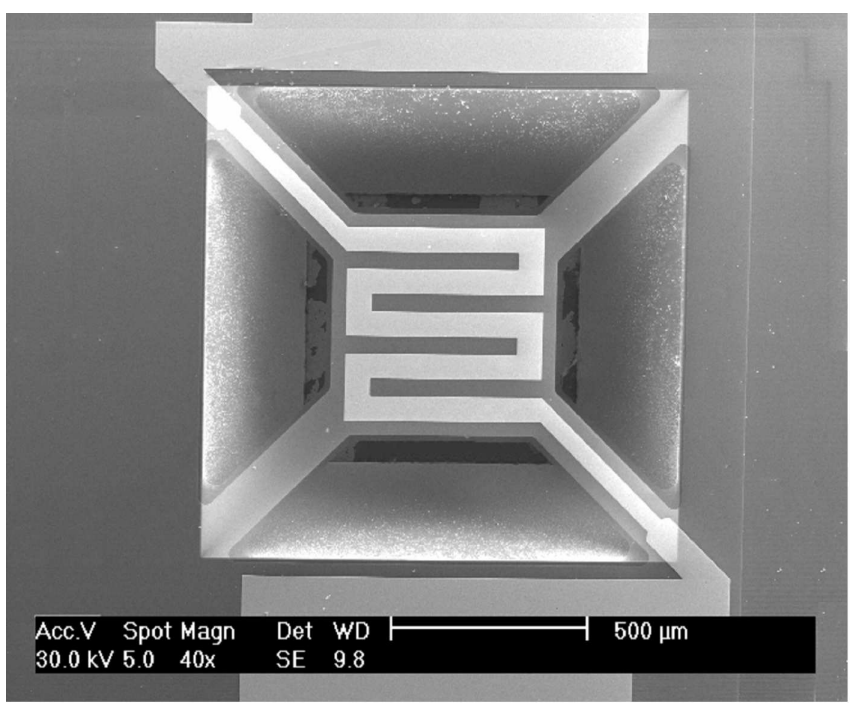

Fig. 2. Scanning electron microscopy (SEM) photograph of the developed thermal sensor.

The flowmeter composed of two such elements $\left(S_{1}\right)$ and $\left(S_{2}\right)$ is placed in the channel connecting two chambers of the detector. The electrical current flowing through the arms of the bridge heats the elements to a temperature of approximately $150^{\circ} \mathrm{C}$. As a result of the gas flow the first element is cooled down while the second, around which the gas heated on the first element flows, heats up. This causes a change in their resistance and creation of a signal on the output of the differential amplifier. Figure 3 shows the temperature around the elements for two conditions, without flow $(Q=0)$ and with flow $(Q>0)$.

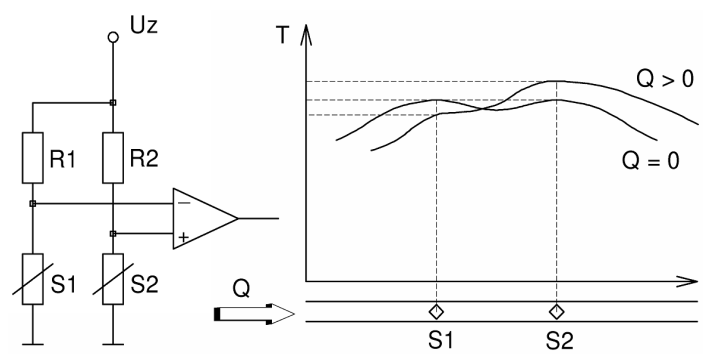

Fig. 3. (a) Scheme of the electric circuit and (b) the principle of the flowmeter operation.

Quantitative analysis of phenomena occurring in thermal anemometers is a very complex task. In our case, the theoretical calculation of the anemometric characteristics was abandoned because of the complicated shape of the elements. Instead, a semi-empirical model of the flowmeter operation, which make possible to optimize the conditions of its work, was developed. The frequency characteristics, which have significant influence on the operation of an optopneumatic detector, were also measured.

\section{Anemometer characteristics}

The flow measurement bases on convective heat transfer from the hot element to its gas environment [7-9]. In the simplest form, convective heat transfer can be expressed as

$$
P=W\left(T-T_{0}\right),
$$

where $P$ - electric power released in hot-film equal to heat transfer rate, $W$ - convective heat transfer coefficient between the element and the fluid, $T$ - mean element temperature, $T_{0}$ - ambient temperature. The coefficient $W$ is a complex function of the flow rate $Q$ and the character of the flow, fluid parameters and the shape of the element. In practice, it has to be determined experimentally for a single flowmeter system. For the tested system the values of heat transfer coefficients are shown in Fig. 4.

The presented relationships $W(Q)$ are significantly flattened at the initial section. It is connected with natural convection of the gas rounding the heated element, which is noticeable at slow forced flow. In this case the real flow rate around the element is higher than the flow calculated when the rate at which the gas is being delivered to the system is taken into account.

The optimization of working parameters of the flowmeter, presented in Fig. 5, requires the knowledge of mutual 


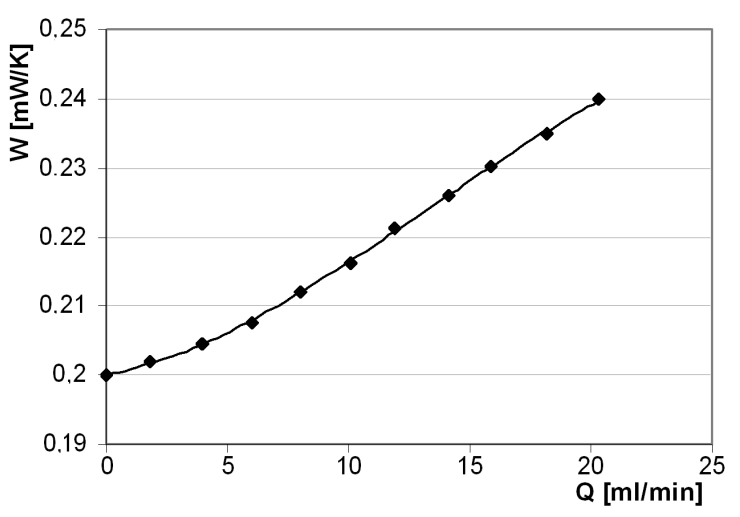

Fig. 4. The heat transfer coefficient for the selected element.

thermal influence of both elements. First, changes of the temperature of element $S_{2}$, during electrical heating of element $S_{1}$, at different gas flow rates and at different ambient temperatures were examined.

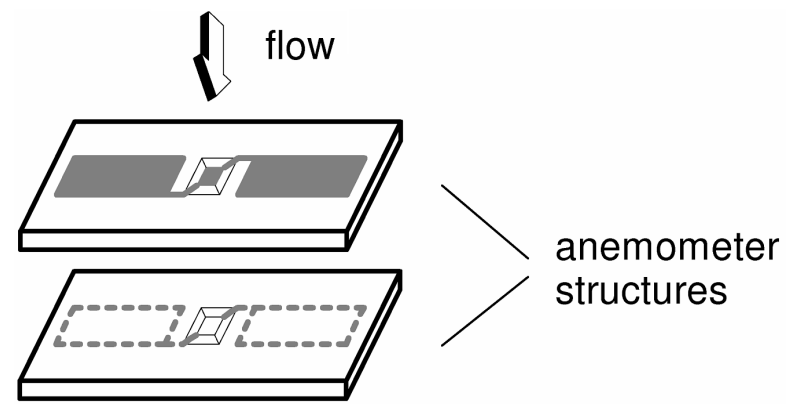

Fig. 5. The assembly of the flowmeter.

It was found out that the increase of temperature of element $S_{1}$ causes linear increase of the temperature of the neighbouring element which is in equilibrium with the surroundings, regardless of the direction and the value of the gas flow rate. It is shown in Fig. 6 .

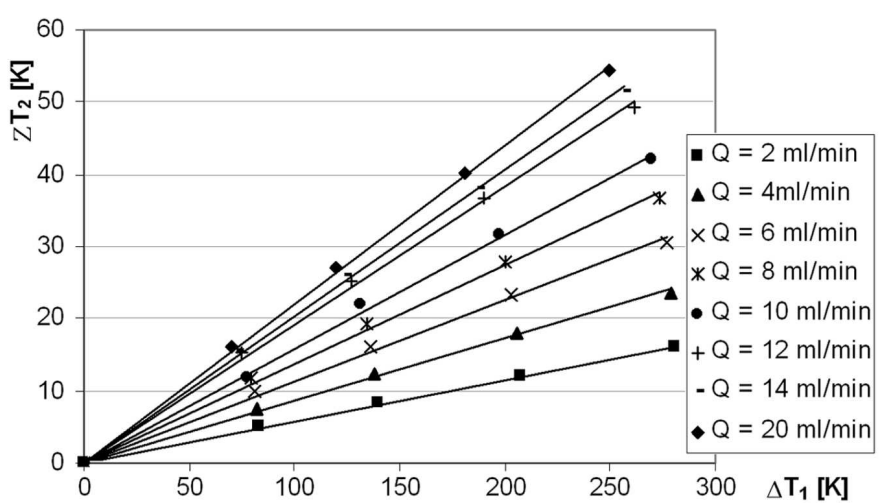

Fig. 6. The increase in temperature of element $S_{2}$ as a function of an increase in temperature of element $S_{1}$ for selected values of the gas flow rate.
When both elements are heated at the same time, their interaction is more complicated. For each element the influence of the neighbour is similar to the changes in the ambient temperature, which is a function of the gas flow rate. The distribution of the temperatures for both elements versus flow rate is presented in Fig. 7. An increase of the flow rate is accompanied by an increase in temperature of the element, which is flown round by the gas warmed up on the first element. However, after exceeding certain limit (about $5 \mathrm{ml} / \mathrm{min}$ ), the temperature of both elements decreases with increasing the flow rate.

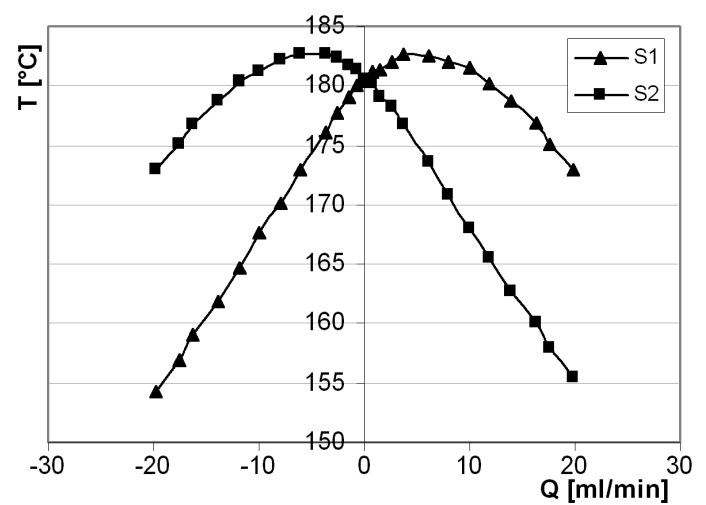

Fig. 7. The distribution of the element temperatures versus flow rate.

Comparing the differential signal with the characteristics for single elements (Fig. 8), the possibility to distinguish the flow direction and the existence of a straight-line characteristic in the vicinity of zero flow attracted our attention. However, the measuring range of the flowmeter becomes narrower, because the signal has unequivocal values only for the flows from a range of about -20 to about $20 \mathrm{ml} / \mathrm{min}$. Still it is of no importance for optopneumatic detectors, where the flow is not higher than $1 \mathrm{ml} / \mathrm{min}$. The existence of a constant component of signal for $Q=0$ is a result of different nominal resistance of the elements, which causes a loss of bridge symmetry. For small differences in the resistance it is of no significance because in the case of optopneumatic detectors only the variable component of a signal is taken into account.

Analyzing the sensitivity of the investigated system $\mathrm{d}(\Delta U) / \mathrm{d} Q$ it can be concluded that the flowmeter shows the maximum of its sensitivity in the vicinity of $Q=0$, while in the case of a classic system with one element a considerable loss in sensitivity in the region of small flows is observed. It is a result of natural gas convection around the heated element. The absolute value of the sensitivity in both systems is of the same order. The influence of the ambient temperature is comparable and manifests itself in a comparatively slight decrease of the sensitivity with an increase of temperature.

The frequency characteristic of a flowmeter is the next important factor for its use in an optopneumatic detector. In this paper, the static model of flowmeter oper- 


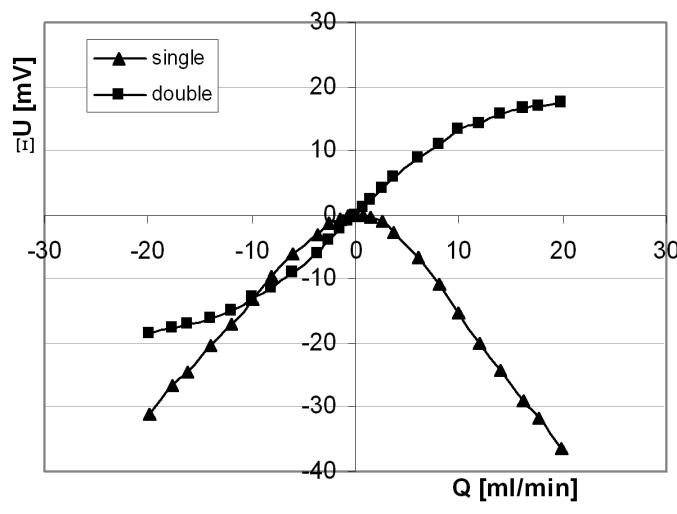

Fig. 8. The voltage changes for flowmeters containing one and two thermo anemometric elements.

ation was considered. However, it can be also used for slow-variable courses. A limit frequency, according to the model, amount to $1 / \tau_{\mathrm{e}}$, where $\tau_{\mathrm{e}}$ - time constant of an element. The time constants of the investigated elements are of an order of $10 \mathrm{~ms}$. This guarantees an appropriate work dynamics of the presented flowmeter in the detectors for which the modulation frequency of radiation is not higher than $100 \mathrm{~Hz}$. These values are much smaller than for hot-wire anemometers and are comparable with other flowmeters with film sensors. The increase in the system dynamics is still possible by using constants temperature system, e.g. similar to the one presented in [10] and appropriate signal processing.

\section{Summary}

The constructed flowmeter was applied successfully in the optopneumatic detectors used for measuring the concentration of gases such as $\mathrm{SO}_{2}, \mathrm{CO}, \mathrm{CO}_{2}$ and $\mathrm{C}_{n} \mathrm{H}_{m}$. It is characterized by high durability, which is a result of a low working temperature (less than $150^{\circ} \mathrm{C}$ above the surroundings temperature) and conducting paths considerably thicker than that of typical hot wire anemometers. The differential mode of work employed in this flowmeter make possible to distinguish the flow direction and to obtain high sensitivity at the cost of the measuring range, which is not important in the case of optopneumatic detectors. The use of flow measurement (instead of pressure) for signal generation also eliminates mechanical and acoustic disturbances, common when microphones are used.

The optimal operating conditions exist when both elements have similar nominal resistance. This results in symmetric anemometric characteristics for the flows in the opposite directions and the minimal influence of the surroundings temperature on the measurement results.

Further development of the flowmeter should include the use of constant temperature system, which will result in better dynamics, the optimization of the distance between the elements and the optimization of the elements dimensions. Especially, the pneumatic resistance of a flowmeter should be taken into consideration, because it is very important parameter affecting the operation of the optopneumatic detector.

\section{References}

[1] J.A. Jahnke, Continuous Emission Monitoring, Van Nostrand Reinhold, New York 1993.

[2] W. Jakubik, M. Urbanczyk, E. Maciak, T. Pustelny, Bull. Pol. Acad. Sci. 56, 133 (2008).

[3] K. Golaszewska, E. Kaminska, T. Pustelny, P. Struk, T. Piotrowski, A. Piotrowska, M. Ekielski, R. Kruszka, M. Wzorek, M. Borysewicz, I. Pasternak, K. Gut, Acta Phys. Pol. A 114, A-221 (2008).

[4] Gas Analysis Engineering Equipment and Systems, Siemens, Catalogue PA 10, 1993.

[5] K. Jasek, Ph.D. Thesis, Military University of Technology, Warsaw 1998 (in Polish).

[6] K. Jasek, B. Mazurek, M. Pasternak, J. Phys. IV (France) 129, 125 (2005).

[7] H. Bruun, Hot-Wire Anemometry Principles and Signal Analysis, Oxford University, Cambridge 1993.

[8] A. Szpakowski, C. Tyszkiewicz, T. Pustelny, Acta Phys. Pol. A 114, A-237 (2008).

[9] Z. Biernacki, Sensors and Thermoanenometric Systems, WKiŁ, Warszawa 1997 (in Polish).

[10] J. Kiełbasa, Z. Smolarski, Bull. Acad. Pol. Sci. XXVI, 10 (1978). 